\title{
FUNCTIONAL PROPERTIES OF THE SOLUTIONS OF DIFFERENTIAL SYSTEMS*
}

BY

WILLIAM M. WHYBURN

Numerous contributions have recently been made to the subject of functionals. The applications of work in this field are found in many branches of pure mathematics and mathematical physics. Bliss $\dagger$ developed functional properties of the solutions of a system of differential equations and made use of these properties in his work on ballistics. In the new field of wave mechanics a tool that is of extreme usefulness is a method of perturbations based entirely upon underlying functional properties of the solutions of differential equations. An investigation of stability in dynamical systems, especially stability of the perturbative type, $\downarrow$ is essentially a study of the functional properties of a class of differential systems. Recently W. L. Hart $\$$ has developed the notion of continuity for a functional of arguments that are summable functions of their variables.

The present paper considers a general class of systems of non-linear differential equations of the first order and shows that the solutions of these systems are uniformly continuous functionals of the non-linear functions and the initial values in a stronger sense than is ordinarily considered. Estimates are given for the magnitudes of the variations in the solutions in terms of the variations of the quantities upon which they depend. All of the variables and functions in the paper are supposed real.

Consider the system of ordinary differential equations

(1) $d y_{i} / d x=f_{i}\left(x, y_{1}, \cdots, y_{n}\right)+\sum_{j=1}^{i=n} A_{i j}\left(x, y_{1}, \cdots, y_{n}\right) y_{j}(i=1, \cdots, n)$, where the following conditions are imposed:T

A. For each fixed $x$ on $X: a \leqq x \leqq b$, the functions $f_{i}(x, y)$ and $A_{i j}(x, y)$ are continuous in $\left(y_{1}, \cdots, y_{n}\right)$ for all real values of $y_{1}, \cdots, y_{n}$.

B. For each fixed $\left(y_{1}, \cdots, y_{n}\right)$, the functions $A_{i j}(x, y)$ and $f_{i}(x, y)$ are summable in $x$ on $X$.

* Presented to the Society, June 21, 1929; received by the editors September 3, 1929.

$\dagger$ These Transactions, vol. 21 (1920), pp. 79-92, and p. $92 \mathrm{ff}$.

† See Birkhoff, American Journal of Mathematics, vol. 49 (1927), pp. 1-38.

$\S$ Annals of Mathematics, (2), vol. 24 (1922-23), pp. 23-38.

ๆ $A_{i j}(x, y)=A_{i j}\left(x, y_{1}, \cdots, y_{n}\right), f_{i}(x, y)=f_{i}\left(x, y_{1}, \cdots, y_{n}\right)$, etc. 
C. There exist summable functions $M(x)$ and $L(x)$ on $X$ such that for all $x$ on $X$ and for all real $\left(y_{1}, \cdots, y_{n}\right),\left(z_{1}, \cdots, z_{n}\right)$

$$
\begin{gathered}
\left\{\begin{array}{l}
\left|f_{i}(x, y)\right| \\
\left|A_{i j}(x, y)\right|
\end{array}\right\} \leqq M(x), \\
\left\{\begin{array}{l}
\left|A_{i j}(x, y)-A_{i j}(x, z)\right| \\
\left|f_{i}(x, y)-f_{i}(x, z)\right|
\end{array}\right\} \leqq L(x) \sum_{r=1}^{r-n}\left|y_{r}-z_{r}\right| \quad(i, j=1, \cdots, n) .
\end{gathered}
$$

The author has recently shown* that if $T$ is an arbitrarily given constant and if $\alpha_{1}, \cdots, \alpha_{n}, a_{1}, \cdots, a_{n}$ are constants such that

D. $\left|\alpha_{i}\right|<T, i=1, \cdots, n$, and the points $x=a_{i}, i=1, \cdots, n$, all lie on a single sub-interval $\Gamma$ of $X$ of length $\dagger \delta$, then there exists a unique solution of (1) which satisfies

$$
y_{i}\left(a_{i}\right)=\alpha_{i} \quad(i=1, \cdots, n)
$$

where by a solution of (1) we understand a set of absolutely continuous functions $y_{1}(x), \cdots, y_{n}(x)$ that satisfies (1) almost everywhere on $X$. This existence theorem is quite general and includes the usual existence and uniqueness theorems for systems of the type ordinarily considered, i.e., where $A_{i j}=0, a_{1}=a_{2}=\cdots=a_{n}$, as special cases and in addition covers other systems, e.g., the linear system. We treat this more general system (1), (2) in such a way that the results for the ordinary systems occur as special cases of our results.

For a given $M(x), L(x)$, and $T$ let $Q$ denote the class of functions $A_{i j}(x, y)$, $f_{i}(x, y), \alpha_{i}, a_{i}, i, j=1, \cdots, n$, that satisfy conditions A, B, C, D. Let $R$ denote the sub-class of $Q$ for which $a_{1}=a_{2}=\cdots=a_{n}=c$.

Theorem I. For given $M(x), L(x)$, and $T$, there exists a constant $B$ such that if $A_{i j}, f_{i}, \alpha_{i}, a_{i}, i, j=1, \cdots, n$, are any functions $\ddagger$ of $Q$ and $y_{1}(x), \cdots$, $y_{n}(x)$ is the unique solution of (1), (2), then

$$
\left|y_{i}(x)\right| \leqq H \quad(i=1, \cdots, n, a \leqq x \leqq b) .
$$

An examination of the author's paper $\S$ shows that the quantity $G$ there determined depends only on $T, L$, and $M$-the constants $r$ and $R$ having previously assigned constant values-and is therefore a constant for all $A_{i j}, f_{i}, \alpha_{i}, a_{i}$, in $Q$. When we choose a point $x=c$ of $\Gamma$ and examine the lemma of Theorem I of our previous paper, $\mathbb{T}$ we see that if $K$ of that lemma is chosen

* Annals of Mathematics, (2), vol. 30 (1928), pp. 31-38.

$\dagger \delta$ is expressed in terms of $L, M$, and $T$ in the paper referred to.

$\ddagger$ In case $A_{i j} \equiv 0, i, j=1, \cdots, n, a_{1}=a_{2}=\cdots=a_{n}$, this theorem follows directly from an application of Theorem IV of the present paper.

$\S$ Loc. cit., p. 35, Lemma 2 of Theorem II.

I Loc. cit., p. 32. 
greater than $n G+\int_{a}^{b} M(t) d t$, we obtain a constant $H=K \exp \left[n \int_{a}^{b} M(t) d t\right]$ such that for any given set of quantities $A_{i j}, f_{i}, \alpha_{i}, a_{i}$, in $Q$, we have

$$
\left|y_{i}{ }^{(k)}(x)\right| \leqq H \quad(i=1, \cdots, n, k=0,1, \cdots, a \leqq x \leqq b) .
$$

Since $y_{i}(x)$ is the limit of the sequence of functions $y_{i}{ }^{(k)}(x), k=0,1, \cdots$, it follows that $\left|y_{i}(x)\right| \leqq H, i=1, \cdots, n, a \leqq x \leqq b$. Theorem I follows from the observation that $H$ is independent of the particular set of quantities $A_{i j}$, $f_{i}, \alpha_{i}, a_{i}$, of $Q$ that is used.

THEOREM II. Let $u_{1 j}(x), \cdots, u_{n j}(x), j=1, \cdots, n$, be the solutions of

$$
d u_{i} / d x=\sum_{j=1}^{j=n} F_{i j}(x) u_{j}, u_{i r}(c)=\left\{\begin{array}{ll}
0, & i \neq r \\
1, & i=r
\end{array} \quad(i=1, \cdots, n),\right.
$$

where $x=c$ is a point of $X, F_{i j}(x)$ is summable on $X$, and let $J(x)$ be a summable function of $x$ on $X$ such that $\left|F_{i j}(x)\right|<J(x), i, j=1, \cdots, n$. Let $W(x)$ be the determinant whose element in the ith row and jth column is $u_{i j}(x)$ and let $\beta_{i j}$ be the cofactor of $u_{i j}$ in $W$. There exists a quantity $N$ which is a constant for a given $J(x)$ and is such that if $K_{i j}=\beta_{i j} / W$, then $\left|K_{i j}\right|<N(i, j=1, \cdots, n)$, $a \leqq x \leqq b$, and for all summable functions $F_{i j}(x)$ such that $\left|I_{i j}(x)\right|<J(x)$ on $X$.

A well known theorem gives $W(x)=\exp \left[\int_{c x} \sum_{n} 1 F_{i i}(t) d t\right]$. Since $\left|F_{i i}(t)\right|<J(t)$ on $X$, we have $W(x)>\exp \left[-\int_{a}^{a} n J(t) d t\right]>0$. Applying Theorem I to $u_{i j}(x)$, we get it less than a constant $S$, independent of $F_{i j}$, and hence each element of the determinant $\beta_{i j}$ is less than $S$. An application of Hadamard's theorem* yields $\beta_{i j} \leqq\left(n S^{2}\right)^{n / 2}$, hence $\left|K_{i j}(x)\right|<N$, where

$$
N=\left(S^{2} n\right)^{n / 2} \exp \left[\int_{a}^{b} n J(t) d t\right] .
$$

Theorem III. Let $F(x), f_{i}(x), g_{i}(x), i=1, \cdots, n$, be finite-valued and measurable functions on the measurable point set $E$ and let $0 \leqq F(x) \leqq \sum_{i=1}^{n}$ $f_{i}(x) g_{i}(x)$ hold on $E$. There exist measurable functions $h_{1}(x), \cdots, h_{n}(x)$ such that $\left|h_{i}(x)\right| \leqq\left|g_{i}(x)\right|$ and $F(x)=\sum_{i=1}^{n} f_{i}(x) h_{i}(x)$ on $E$.

Let $K_{0}$ be the subset of $E$ on which $F(x)=0$ and let $K_{i}, i=1, \cdots, n$, be the subset of $E$ on which $0, f_{1} g_{1}, f_{1} g_{1}+f_{2} g_{2}, \cdots, \sum_{j=1}^{i-1} f_{i} g_{j}<F(x) \leqq \sum_{j=1}^{i}$ $f_{i} g_{j}$. The sets $K_{i}$ and $K_{i}, i \neq j$, have no common points, $g_{i}(x) f_{i}(x)$ is positive on $K_{i}$ for each $i=1, \cdots, n$, and $E=\sum_{j=0}^{n} K_{j}$. Let $h_{1}(x) \equiv 0$ on $K_{0}, h_{1} \equiv F(x)$ - $\left[f_{1}(x)\right]^{-1}$ on $K_{1}$, and $h_{1} \equiv g_{1}$ on $\sum_{j=2}^{n} K_{j}$. Let $h_{i}(x)$ be zero on $\sum_{j=0}^{i-1} K_{j}$, $h_{i}=\left[F(x)-\sum_{j=1}^{i-1} f_{j} g_{j}\right] / f_{i}(x)$ on $K_{i}$, and $h_{i}(x) \equiv g_{i}(x)$ on $\sum_{j=i+1}^{n} K_{j}(i=2$, $\cdots, n)$.

* See Lovitt, Linear Integral Equations, New York, 1924, p. 31. 
The construction shows immediately that $\left|h_{i}(x)\right| \leqq\left|g_{i}(x)\right|$ and $F(x)$ $=\sum_{i=1}^{n} f_{i} h_{i}$ on $E$. Each of the point sets $K_{i}$ is measurable since $K_{0}$ is measurable and the measurability of $K_{j}$ follows from that of $K_{0}, \cdots$, $K_{j-1}$. For $K_{j}$ is the subset of the measurable set $E-\sum_{i=0}^{j-1} K_{i}$ on which the measurable function $\sum_{i=1}^{j} f_{i} g_{i}-F(x)$ is positive or zero. Since $f_{i}(x) \neq 0$ on $K_{i}$, we may apply a well known theorem* concerning products and quotients of measurable functions to get that $h_{i}$ is measurable on $K_{i}$. On $\sum_{j=i+1}^{n} K_{j}$, $h_{i}$ is equal to the measurable function $g_{i}(x)$ while $h_{i} \equiv 0$ on $\sum_{j=0}^{i-1} K_{j}$. Hence $h_{i}$ is measurable on $E$.

Corollary 1. If $F(x)=|A(x)|$, where $A(x)$ is measurable on $E$, then $A(x)=\sum_{i=1}^{n} H_{i}(x) f_{i}(x)$, where $H_{i}(x)=+h_{i}(x)$ or $-h_{i}(x)$ according as $A(x) \geqq 0$ or $A(x) \leqq 0$. Hence $H_{i}(x)$ is measurable and $\left|H_{i}(x)\right| \leqq\left|g_{i}(x)\right|$ on $E$.

COROLlaRy 2. If the functions $g_{i}(x)$ are summable on $E$, the functions $h_{i}(x)$ and $H_{i}(x)$ of Theorem III and Corollary 1 are summable on E. $\dagger$

TheOREM IV. Let $f(x)$ be defined on any interval $X$ and let $M$ denote the subset of $X$ on which $d f / d x$ exists (either finite or infinite with definite sign); then with the possible exception of a countable set of points $d|f(x)| / d x$ exists and $|d| f(x)|/ d x|=|d f(x) / d x|$.

Let $x=p$ be any point of $M$ for which $f(p) \neq 0$. Since $f^{\prime}(p)$ exists, $f(x)$ is continuous at $x=p$ and there exists a neighborhood of $x=p$ throughout which $f(x) f(p)>0$. Throughout this neighborhood we have either $|f(x)|$ $=f(x)$ or $|f(x)|=-f(x)$. In either case $d|f(p)| / d x$ exists and is numerically equal to $\left|f^{\prime}(p)\right|$.

Let $x=p$ be a point of $M$ at which $f(x)=0$. From the existence of $f^{\prime}(p)$ we have $\lim _{h \rightarrow p^{+}} f(h) /(h-p)=f^{\prime}(p)$ and hence $\lim _{h \rightarrow p^{-}}|f(h)| /(h-p)=$ $-\left|f^{\prime}(p)\right|, \lim _{h \rightarrow p^{+}}|f(h)| /(h-p)=\left|f^{\prime}(p)\right|$. Hence both the left-hand and right-hand derivatives of $|f(x)|$ exist at every point of $M$. A theorem of G. C. Young $\ddagger$ may now be applied to show that these two derivatives differ from each other at most at a countable set of points of $M$.

COROLlaRy. There does not exist a function that is defined on an interval and vanishes at more than a countable set of points at each of which its derivative exists (either finite or infinite with definite sign) and is different from zero. $\$$

* See Carathéodory, Vorlesungen ueber reelle Funktionen, Leipzig, 1928, p. 378.

$\uparrow$ This follows since these functions are measurable and bounded numerically by the summable functions $\left|g_{i}(x)\right|$. See Carathéodory, loc. cit., p. 442.

$\ddagger$ For a statement of this theorem, see Hobson, Theory of Functions of a Real Variable, Cambridge University Press, second edition, vol. I, pp. 368 and 369. The consequence of Young's theorem that applies directly in the above proof is stated at the bottom of p. 369 .

$\S$ This corollary follows from Theorem IV and its proof when we observe that the derivative of $|f(x)|$ does not exist at a point where $f(x)$ vanishes and has a derivative that is different from zero. 
Equations (1), (2) define $y_{1}(x), \cdots, y_{n}(x)$ as functionals of $f_{i}(x, y)$, $A_{i j}(x, y), \alpha_{i}, a_{i}(i, j=1, \cdots, n)$, in $Q X$ in the sense that for each set of functions in $Q$, the system (1), (2) determines unique absolutely continuous functions $y_{1}, \cdots, y_{n}$ on $X$ that satisfy (2) and satisfy (1) almost everywhere.

Following the definitions given by W. L. Hart, ${ }^{*}$ we say that the functions $y_{r}(x)(r=1, \cdots, n)$ are continuous functionals of $A_{i j}, f_{i}, \alpha_{i}, a_{i}$ at $B_{i j}, g_{i}$, $\beta_{i}, b_{i}$ of $Q$ if for every given positive number $\epsilon$, there exists a positive number $\delta_{e}$, such that if $A_{i j}, f_{i}, \alpha_{i}, a_{i}$ belong to $Q$ and $\left|a_{i}-b_{i}\right|<\delta_{e},\left|\alpha_{i}-\beta_{i}\right|<\delta_{e}$, $\int_{a}\left|f_{i}(x, u)-g_{i}(x, u)\right| d x<\delta_{\epsilon}, \int_{a}^{b}\left|A_{i j}(x, u)-B_{i j}(x, u)\right| d x<\delta_{\epsilon}$, for all absolutely continuous functions $u_{1}(x), \cdots, u_{n}(x)$ that are bounded numerically on $X$ by $H$, the constant determined in Theorem I, $i, j=1, \cdots, n$, then $\mid y_{r}(x)$ $-z_{r}(x) \mid<\epsilon$ for every $x$ on $X$, where $y_{1}(x), \cdots, y_{n}(x)$ and $z_{1}(x), \cdots, z_{n}(x)$, fespectively, are the solutions of system (1), (2) corresponding to $A_{i j}$, $\mathrm{r}_{i}, \alpha_{i}, a_{i}$, and $B_{i j}, g_{i}, \beta_{i}, b_{i}$. The continuity is said to be uniform in $Q$ if the number $\delta_{i}$ can be chosen independent of the functions $B_{i j}, g_{i}, \beta_{i}, b_{i}$, so long as they belong to $Q$.

Theorem V. The solution functions, $y_{1}(x), \cdots, y_{n}(x)$, of system (1) and

$$
y_{i}(c)=\alpha_{i}, \quad a \leqq c \leqq b \quad(i=1, \cdots, n),
$$

are uniformly continuous functionals of $A_{i j}, f_{i}, \alpha_{i}$, in $X R$.

Let a positive number $\epsilon$ be assigned and choose $\delta_{\epsilon}$ so that

$$
\delta_{.} \leqq \epsilon /[n S\{1+n N(1+n H)\}],
$$

where $N$ and $S$ are the constants whose existence was demonstrated in Theorem II for $F_{i j}(x) \equiv L(x)+M(x)+n H L(x)$ and $H$ is the constant whose existence is proved in Theorem I. Let $A_{i j}, f_{i}, \alpha_{i}$, and $B_{i j}, g_{i}, \beta_{i}$, be two sets of functions in $R$ and meeting the requirements of our definition of continuity with respect to $\delta_{c}$. Let $y_{i}(x)$ and $z_{i}(x)$, respectively, be the corresponding solutions of (1), (4), and let $u_{i}(x)=y_{i}(x)-z_{i}(x), i=1, \cdots, n$. Almost everywhere on $X$ we have

$$
\begin{aligned}
u_{i}^{\prime}= & f_{i}(x, y)-g_{i}(x, z)+\sum_{j=1}^{n}\left[A_{i j}(x, y) y_{j}-B_{i j}(x, z) z_{j}\right] \\
= & {\left[f_{i}(x, y)-f_{i}(x, z)\right]+\left[f_{i}(x, z)-g_{i}(x, z)\right]+\sum_{j=1}^{n}\left\{\left[A_{i j}(x, y)\right.\right.} \\
& \left.\left.-B_{i j}(x, y)\right] y_{j}(x)+\left[B_{i j}(x, y)-B_{i j}(x, z)\right] y_{j}(x)+B_{i j}(x, z)\left[y_{j}-z_{j}\right]\right\} .
\end{aligned}
$$

Taking absolute values and making use of the inequalities of condition $\mathbf{C}$ and Theorem I, we have

* Loc. cit., p. 25 . 


$$
\left|u_{i}^{\prime}\right| \leqq[L(x)+M(x)+B n L(x)] \sum_{r=1}^{n}\left|u_{r}\right|+\phi_{i}(x) \quad(i=1, \cdots, n),
$$

where

$$
\phi_{i}(x)=\left|f_{i}(x, z)-g_{i}(x, z)\right|+H \sum_{j=1}^{n}\left|A_{i j}(x, y)-B_{i j}(x, y)\right|
$$

and $y_{i}(x)$ and $z_{i}(x)$ are absolutely continuous functions of $x$ on $X$. Replacing $\left|u_{i}\right|$ by $U_{i}$ and making use of Theorem IV, we have

$$
\left|U_{i}^{\prime}\right| \leqq[L(x)+M(x)+n H L(x)] \sum_{r=1}^{n} U_{r}+\phi_{i}(x),
$$

almost everywhere on $X$. Application of Theorem III and its corollaries yields

$$
U_{i}^{\prime}=C_{i}(x) \sum_{r=1}^{n} U_{r}+\theta_{i}(x), \quad U_{i}(c)=\left|\alpha_{i}-\beta_{i}\right|,
$$

almost everywhere on $X$, where $C_{i}(x)$ and $\theta_{i}(x)$ are summable on $X$ and

$$
\left|C_{i}(x)\right| \leqq[L+M+n H L],\left|\theta_{i}(x)\right| \leqq \phi_{i}(x) .
$$

Solving (6) by a generalization of the method of variation of parameters yields

$$
U_{i}(x)=\sum_{r=1}^{n}\left[\sum_{i=1}^{n} \int_{c}^{x} \theta_{j}(t) K_{j r}(t) d t+\left|\alpha_{r}-\beta_{r}\right|\right] u_{i r}(x) \quad(i=1, \cdots, n),
$$

where $u_{i j}(x)$ and $K_{i j}(x)$ are the quantities that occur in Theorem II. Applying Theorem II

$$
\begin{aligned}
U_{i}(x) & \leqq S n N \sum_{j=1}^{n} \int_{c}^{x} \theta_{j}(t) d t+S \sum_{r=1}^{n}\left|\alpha_{r}-\beta_{r}\right| \\
& <S n^{2} N[1+n H] \delta_{\epsilon}+n S \delta_{\epsilon} \leqq S n[1+n N(1+n H)] \epsilon /\{S n[1+n N(1+n H)]\} \\
& <\epsilon,
\end{aligned}
$$

which establishes the desired uniform continuity of $y_{i}(x), i=1, \cdots, n$.

Corollary. The conclusions of Theorem $\mathrm{V}$ are valid for any solution of (1), (4) since $T$ may be chosen as large as one desires.

In applications of functional properties of the solutions of (1), (4) it is of fundamental importance to be able to estimate the magnitude of the change in the solution functions, $y_{i}(x)$, that is produced by a change of known magnitude in $A_{i j}, f_{i}, \alpha_{i}$. The following theorem which is established im- 
mediately by an examination of the proof of Theorem $\mathrm{V}$ gives such an estimate:*

Theorem VI. Let $A_{i j}, f_{i}, \alpha_{i}$ and $B_{i j}, g_{i}, \beta_{i}$ be two sets of functions in $R$ such that $\left|\alpha_{i}-\beta_{i}\right|<\delta, \int_{a}^{b}\left|A_{i j}(x, u)-B_{i j}(x, u)\right| d x<\delta, \int_{a}^{b}\left|f_{i}(x, u)-g_{i}(x, u)\right| d x$ $<\delta$, for all absolutely continuous functions $u_{i}(x)$ on $X$, that are bounded numer $i$ cally by $H, i, j=1, \cdots, n$, and let $y_{1}(x), \cdots, y_{n}(x)$ and $z_{1}(x), \cdots, z_{n}(x)$, respectively, denote the corresponding solutions of (1), (4); then

$$
\left|y_{i}(x)-z_{i}(x)\right|<S n[1+n N(1+n H)] \delta \quad(i=1, \cdots, n),
$$

on $X$, where $S, N$, and $H$ have the same significance as in the proof of Theorem $\mathrm{V}$.

A combination of Theorem $\mathrm{V}$ with the results of our former paper $\dagger$ yields the following more general theorem:

THEOREM VII. The solution functions $y_{1}(x), \cdots, y_{n}(x)$ of system (1), (2) are uniformly continuous functionals of $A_{i j}, f_{i}, \alpha_{i}, a_{i}, i, j=1, \cdots,{ }^{\prime} n$, in $Q X$.

By Theorem $\mathrm{V}$ and its corollary, $y_{n}(x)$ is a uniformly continuous functional on $X$ of $A_{i j}, f_{i}, \gamma_{i}$, where $\gamma_{i}$ is the value of $y_{i}(x)$ at a point $x=c$ of $\Gamma$. By our former theorem, $\ddagger \gamma_{i}$ is a continuous function of $\left(\alpha_{1}, \cdots, \alpha_{n}, a_{1}, \cdots\right.$, $a_{n}$ ) for $a_{j}$ on $\Gamma(i, j=1, \cdots, n)$. It follows from this (by an argument that is entirely analogous to the well known proof that a continuous function of a continuous function is a continuous function) that $y_{r}(x)(r=1, \cdots, n)$ is a continuous functional of $A_{i j}, f_{i}, \alpha_{i}, a_{i}$, in $Q X$. It also follows from this argument and from the observation that our former theorem $\ddagger$ yields uniformity of our type for $\gamma_{r}$ as a functional of $\left(\alpha_{1}, \cdots, \alpha_{n}, a_{1}, \cdots, a_{n}\right)$ that the continuity of the functionals $y_{r}(x)$ is uniform.

* It is to be noted that this estimate can be improved if some of the quantities $A_{i j}, f_{i}, \alpha_{i}$ are fixed. Obvious modifications of the proofs of the paper yield the results here.

† Whyburn, W. M., loc. cit., p. 37, Theorem III.

‡ Loc. cit., p. 37, Theorem III.

The University of California at Los Angeles, Los Angeles, Calif. 